\title{
Migration of intracranial air according to position change in post-traumatic pneumocephalus: erect and supine skull $\mathrm{X}$-ray views
}

\author{
Chan Yong Park ${ }^{1}$, So Ra Ahn ${ }^{1}$, Wu Seong Kang ${ }^{1}$, Hyun Seok Jung ${ }^{2}$, \\ Sang Hyun Seo ${ }^{2}$, Sung Nam Moon ${ }^{2}$ \\ ${ }^{1}$ Department of Trauma Surgery, ${ }^{2}$ Department of Radiology, Wonkwang University, Iksan, \\ Korea
}

Received May 18, 2020

Revised June 22, 2020

Accepted June 22, 2020

Correspondence to

Chan Yong Park

Department of Trauma Surgery,

Wonkwang University Hospital, 895

Muwang-ro, Iksan 54538, Korea

Tel: +82-63-859-2602

Fax: +82-63-859-2029

E-mail:wkafyddl@hanmail.net

\begin{abstract}
Pneumocephalus is often detected in patients with trauma. However, it is difficult to find studies on position change of intracranial air according to the patient position observed on skull X-rays (erect and supine views). This is because skull X-rays are rarely performed in erect position for patients with head injury. We report a case of location change of intracranial air associated with patient position change observed in the skull X-ray of a patient with traumatic pneumocephalus. To the best of our knowledge, no such cases have previously been reported in English literature.
\end{abstract}

Key Words: Trauma, Pneumocephalus, Position, Air, Skull X-ray

\section{Introduction}

Traumatic pneumocephalus is caused by air collection in the intracranial cavity after trauma. When intracranial air causes intracranial hypertension, it leads to tension pneumocephalus, which results in mass effect with neurological deterioration (1-3). Pneumocephalus is generally diagnosed on brain computed tomography (CT) but is rarely identified on skull X-ray, as observed in this case (4).

\section{Case presentation}

A 59-year-old man was referred to our emergency department from another hospital after falling forward from a ladder, on which he was working, at a height of $1.5 \mathrm{~m}$.
He complained of facial pain, had facial swelling, and his Glasgow Coma Scale was 15. Initial vital signs were as follows: blood pressure of $176 / 88 \mathrm{mmHg}$, pulse rate of 87 beats/min, respiration rate of 16 breaths/min, body temperature of $37.4^{\circ} \mathrm{C}$, and oxygen saturation of $94 \%$. Skull $\mathrm{X}$-ray (anteroposterior $[\mathrm{AP}]$ and lateral views) performed in erect position at the previous hospital showed intracranial air in the parietal region (Fig. 1), and fracture of the frontal bone and pneumocephalus were observed on brain CT (Fig. 2). Skull X-ray (AP and lateral views) in supine position and brain CT performed at our hospital showed intracranial air in the frontal region (Fig. 3). Conservative treatment was adjusted, and brain CT performed on the $10^{\text {th }}$ day of admission showed that the intracranial air had nearly disappeared (Fig. 4). The patient was discharged on

(c)This is an Open Access article distributed under the terms of the Creative Commons Attribution Non-Commercial License (http://creativecommons.org/licenses/by-nc/4.0) which permits unrestricted noncommercial use, distribution, and reproduction in any medium, provided the original work is properly cited.

Copyright (C) 2020 Korean Association for Research, Procedures and Education on Trauma. All rights reserved. 


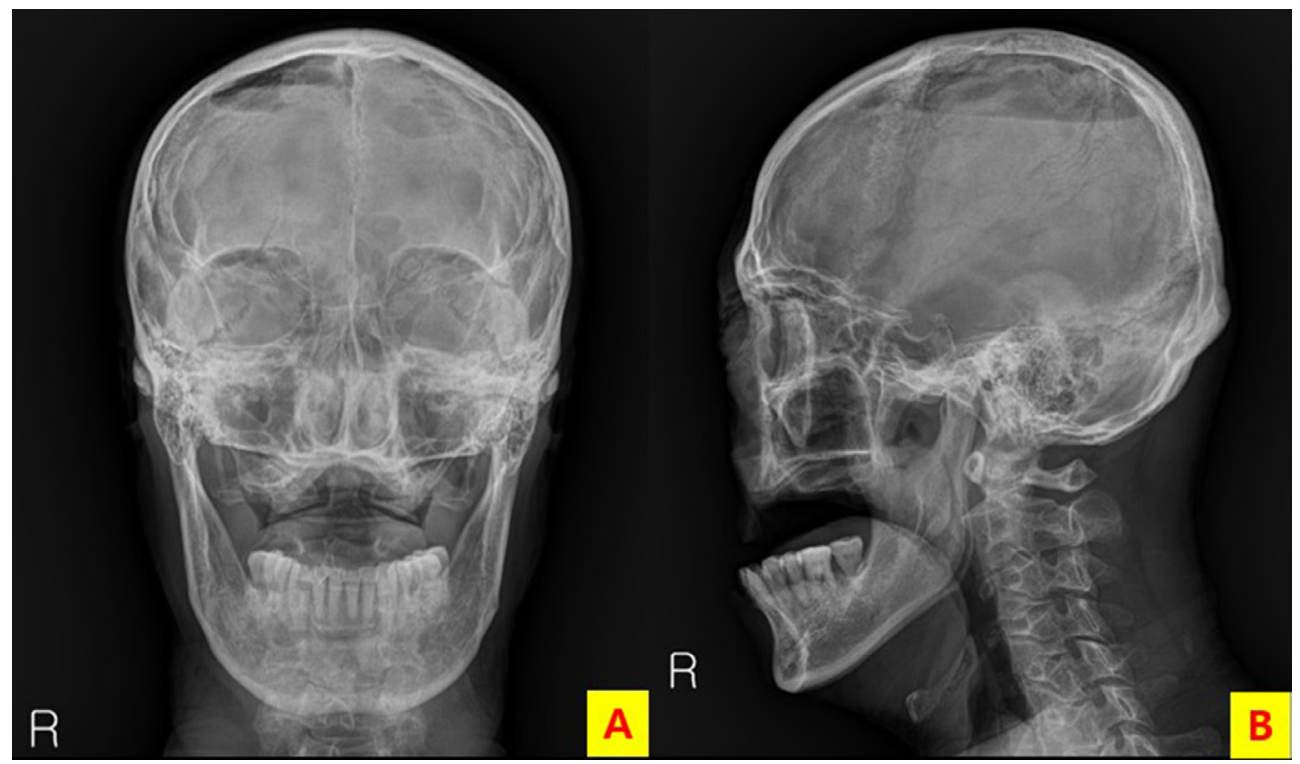

Fig. 1. Skull X-ray (anteroposterior [AP] and lateral views) performed in erect position at a local hospital showing intracranial air in the parietal region.

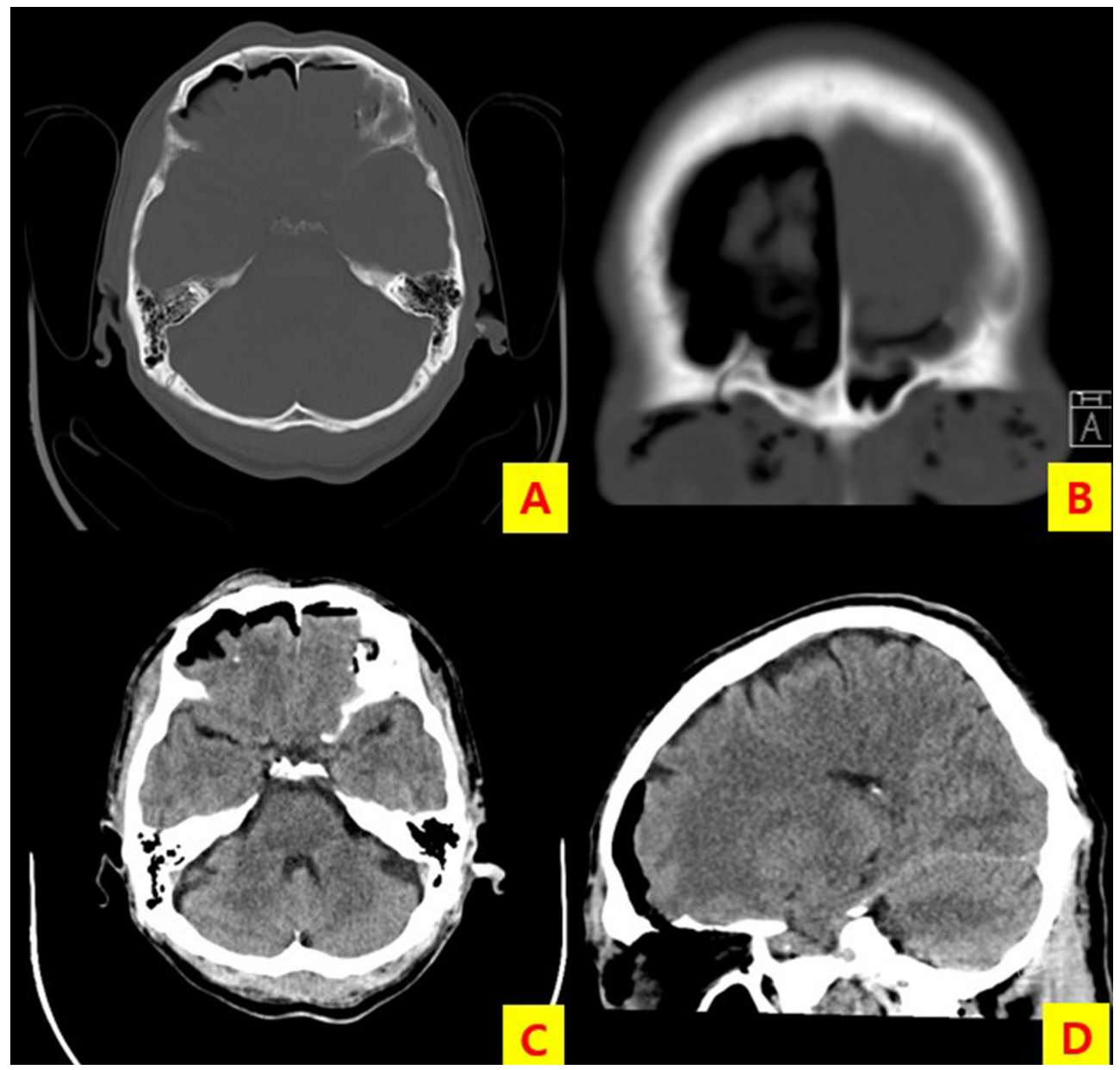

Fig. 2. Brain computed tomography (CT) performed at a local hospital showing fracture and subdural pneumocephalus. 


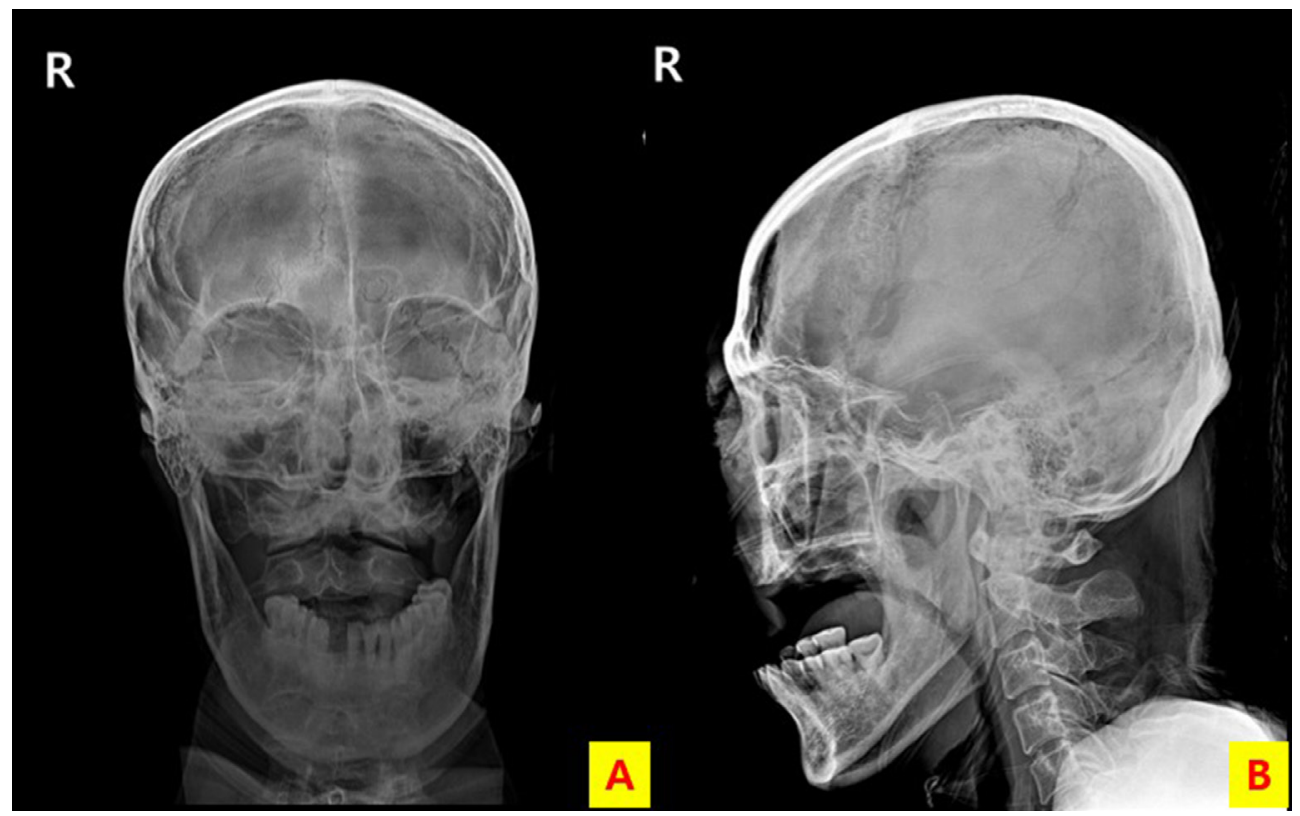

Fig. 3. Skull X-ray (anteroposterior [AP] and lateral views) performed in supine position at our hospital showing intracranial air in the frontal region.

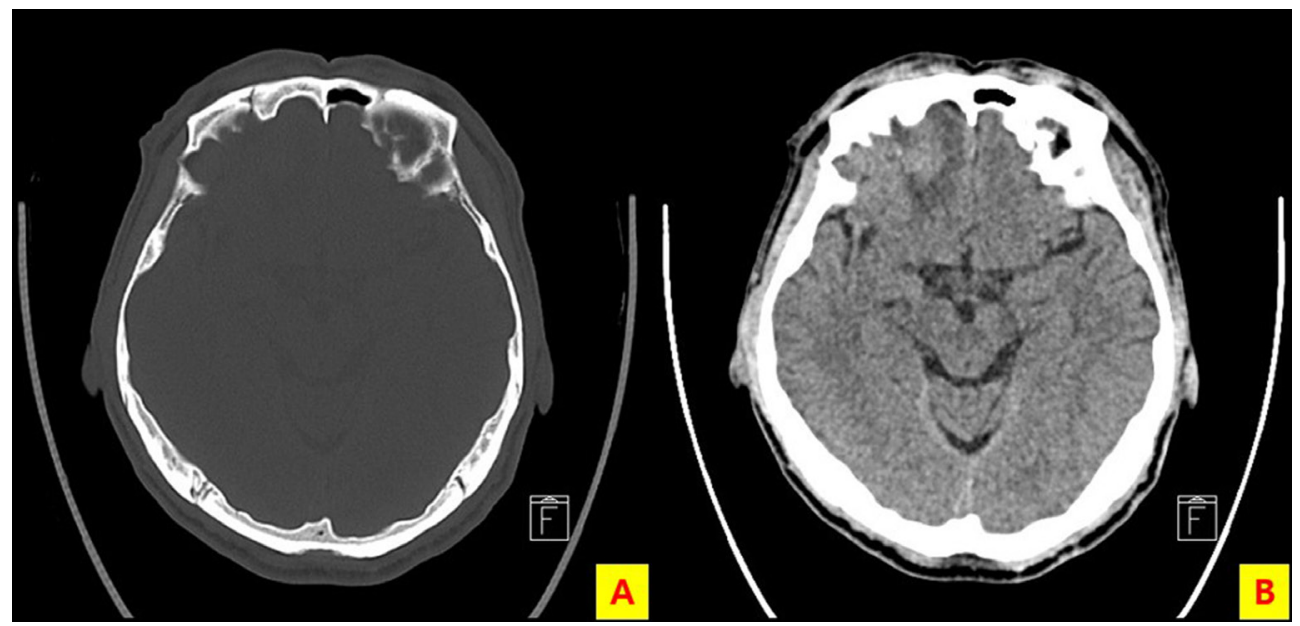

Fig. 4. Brain computed tomography $(\mathrm{CT})$ performed on the $10^{\text {th }}$ day of admission showing nearly complete disappearance of the intracranial air.

the $14^{\text {th }}$ day of admission without any complications .

\section{Discussion}

Traumatic pneumocephalus rarely progresses to tension pneumocephalus, which is accompanied by neurological deterioration and should be considered a neurosurgical emergency (1-3). Pneumocephalus is primarily diagnosed on brain CT and is rarely observed on skull X-ray (4). In this case, skull X-ray was performed in the erect position at another hospital, which showed presence of intracranial air in the parietal region. In contrast, at our hospital, skull X-ray was performed in the supine position, which showed presence of intracranial air in the frontal region. Observing intracranial air migration caused by position change in skull X-rays is extremely difficult because skull $\mathrm{X}$-ray in erect position is rarely performed in case of patients with head injury. To the best of our knowledge, no 
such case has been reported in English literature. In our case, there was no neurological deterioration, conservative treatment was administered, and subsequent brain CT revealed gradual disappearance of the intracranial air.

\section{Conflict of interest}

No potential conflict of interest relevant to this article was reported.

\section{References}

1. Al-Aieb A, Peralta R, Ellabib M, El-Menyar A, Al-
Thani H. Traumatic tension pneumocephalus: Two case reports. Int J Surg Case Rep. 2017;31:145-9.

2. Clement AR, Palaniappan D, Panigrahi RK. Tension Pneumocephalus. Anesthesiology. 2017;127(4):710.

3. Rao V, Fredriksli O, Gulati S. Post-traumatic epidural tension penumocephalus: a case report. J Med Case Rep. 2015;26(9):151.

4. Odebode OT, DunmadeAD, Afolabi OA, Suleiman OA. Lateral skull radiograph in a patient with posttraumatic tension pneumocephalus complicated by late epilepsy. Emerg Med J. 2008;25(1):32. 